\title{
Preliminary Network Centric Therapy for Machine Learning Classification of Deep Brain Stimulation Status for the Treatment of Parkinson's Disease with a Conformal Wearable and Wireless Inertial Sensor
}

\author{
Robert LeMoyne1, Timothy Mastroianni², Donald Whiting3', Nestor Tomycz ${ }^{4}$ \\ ${ }^{1}$ Department of Biological Sciences, Northern Arizona University, Flagstaff, AZ, USA \\ ${ }^{2}$ Cognition Engineering, Pittsburgh, PA, USA \\ ${ }^{3}$ Department of Neurosurgery, AHN Neuroscience Institute, Pittsburgh, PA, USA \\ ${ }^{4}$ AHN Department of Neurosurgery, Pittsburgh, PA, USA \\ Email: robert.lemoyne@nau.edu, rlemoyne07@gmail.com
}

How to cite this paper: LeMoyne, R., Mastroianni, T., Whiting, D. and Tomycz, N. (2019) Preliminary Network Centric Therapy for Machine Learning Classification of Deep Brain Stimulation Status for the Treatment of Parkinson's Disease with a Conformal Wearable and Wireless Inertial Sensor. Advances in Parkinson's Disease, 8, 75-91.

https://doi.org/10.4236/apd.2019.84007

Received: September 28, 2019

Accepted: November 26, 2019

Published: November 29, 2019

Copyright $\odot 2019$ by author(s) and Scientific Research Publishing Inc. This work is licensed under the Creative Commons Attribution International License (CC BY 4.0).

http://creativecommons.org/licenses/by/4.0/

\section{(c) (i) Open Access}

\begin{abstract}
The concept of Network Centric Therapy represents an amalgamation of wearable and wireless inertial sensor systems and machine learning with access to a Cloud computing environment. The advent of Network Centric Therapy is highly relevant to the treatment of Parkinson's disease through deep brain stimulation. Originally wearable and wireless systems for quantifying Parkinson's disease involved the use a smartphone to quantify hand tremor. Although originally novel, the smartphone has notable issues as a wearable application for quantifying movement disorder tremor. The smartphone has evolved in a pathway that has made the smartphone progressively more cumbersome to mount about the dorsum of the hand. Furthermore, the smartphone utilizes an inertial sensor package that is not certified for medical analysis, and the trial data access a provisional Cloud computing environment through an email account. These concerns are resolved with the recent development of a conformal wearable and wireless inertial sensor system. This conformal wearable and wireless system mounts to the hand with the profile of a bandage by adhesive and accesses a secure Cloud computing environment through a segmented wireless connectivity strategy involving a smartphone and tablet. Additionally, the conformal wearable and wireless system is certified by the FDA of the United States of America for ascertaining medical grade inertial sensor data. These characteristics make the conformal wearable and wireless system uniquely suited for the quantification of
\end{abstract}


Parkinson's disease treatment through deep brain stimulation. Preliminary evaluation of the conformal wearable and wireless system is demonstrated through the differentiation of deep brain stimulation set to "On" and "Off" status. Based on the robustness of the acceleration signal, this signal was selected to quantify hand tremor for the prescribed deep brain stimulation settings. Machine learning classification using the Waikato Environment for Knowledge Analysis (WEKA) was applied using the multilayer perceptron neural network. The multilayer perceptron neural network achieved considerable classification accuracy for distinguishing between the deep brain stimulation system set to "On" and "Off" status through the quantified acceleration signal data obtained by this recently developed conformal wearable and wireless system. The research achievement establishes a progressive pathway to the future objective of achieving deep brain stimulation capabilities that promote closed-loop acquisition of configuration parameters that are uniquely optimized to the individual through extrinsic means of a highly conformal wearable and wireless inertial sensor system and machine learning with access to Cloud computing resources.

\section{Keywords}

Parkinson's Disease, Deep Brain Stimulation, Wearable and Wireless Systems, Conformal Wearable, Machine Learning, Inertial Sensor, Accelerometer, Wireless Accelerometer, Hand Tremor, Cloud Computing, Network Centric Therapy

\section{Introduction}

The advent of Network Centric Therapy offers the potential for a quantum leap respective of the treatment of neurodegenerative movement disorders, such as Parkinson's disease. The nascent origins of Network Centric Therapy for providing objective quantification of Parkinson's disease hand tremor derive from the application of wearable and wireless systems, such as the smartphone. The smartphone is equipped with an inertial sensor package that enables the wireless transmission of inertial sensor signal data as an email attachment to the Internet. The email resource serves as a functional Cloud computing resource [1]-[9].

The visionary concept of Network Centric Therapy in the context of neurodegenerative movement disorders, such as Parkinson's disease, pertains to the application of highly wearable and wireless inertial sensor systems that utilize local wireless connectivity to devices with broader wireless accessibility to the Internet, such as a smartphone or tablet. The inertial sensor data package would be conveyed to Cloud computing resources using this segmented wireless strategy. Post-processing resources could be applied from anywhere in the world, which could enable optimal clinical intervention. The neurodegenerative movement disorder, such as Parkinson's disease, could be historically monitored on an effectively continuous basis. Data science technique may further elucidate the 
inherent nature of the neurodegenerative movement disorder. In particular, the robust process of optimizing the parameter configuration for therapy by deep brain stimulation system can be achieved [7] [8] [9].

Recent developments have manifested the opportunities of Network Centric Therapy. The BioStamp nPoint consists of a flexible inertial sensor system that has a profile on the order of a bandage. The BioStamp nPoint may be mounted at a predetermined position on the body for the quantification of movement. The inertial signal data are transmitted by connectivity through connectivity to a device, such as a smartphone. The smartphone with its inherently stronger wireless transmission capability conveys the inertial signal data to a Cloud computing resource for subsequent post-processing [10]. The BioStamp nPoint is the recent evolution of conformal wearable and wireless systems that have been advocated and successfully demonstrated for the capability to quantify characteristics of movement disorder, such as Parkinson's disease [10]-[20].

The research objective is to conduct preliminary Network Centric Therapy for the treatment of Parkinson's disease regarding deep brain stimulation therapy efficacy with the application of machine learning. The BioStamp nPoint mounted about the dorsum of the hand objectively quantifies tremor for Parkinson's disease respective of deep brain stimulation "On" and "Off" status. Based on the quantified inertial sensor signal data the feature set is established through software automation. The multilayer perceptron neural network is selected as the machine learning algorithm to distinguish between the deep brain stimulation "On" and "Off" status. Preliminary Network Centric Therapy for the treatment of Parkinson's disease through deep brain stimulation enables a plausible pathway for the future goal of achieving real-time parameter configuration optimization.

\section{Background}

\subsection{General Perspective of Parkinson's Disease and Conventional Intervention Techniques}

Respective of the United States of America for Parkinson's disease approximately one million people have been diagnosed with this particular movement disorder [21]. The symptoms of Parkinson's disease are notably endemic for people greater than age 55-years-old [22]. A predominant symptom of Parkinson's disease is the presence of a resting tremor [21]. In general, the tremor frequency is on the order of four to five per second [21] [23]. Furthermore, this resting tremor may attenuate or even cease during voluntary movement [23]. The neurological basis for Parkinson's disease is associated with the degeneration of dopaminergic neuron of the substantia nigra [21]. Parkinson's disease symptoms manifest with the progressive decrement of dopamine production for structures of the basal ganglia, such as the caudate and putamen [24].

Traditional therapies for Parkinson's disease involve medication, such as prescribing Levodopa [21] [25] [26]. However, neurosurgery is reserved as an ulti- 
mate intervention, for which medication has been clinically deemed as ineffective [21] [25]. The pallidotomy and thalamotomy neurosurgical are procedures that induce permanent lesioning of deep brain structure pathways [21] [25] [27] [28] [29].

\subsection{Deep Brain Stimulation for the Treatment of Parkinson's Disease and Optimization Challenges}

On the order of three decades ago during the later 1980's Dr. Alim-Louis Benabid successfully demonstrated the concept of deep brain stimulation for the treatment of Parkinson's disease [30] [31] [32]. The deep brain stimulation system consists of electrode leads that are connected to an implantable pulse generator powered by a battery to generate an electrical signal to a targeted structure of the deep brain [33]. Respective of Parkinson's disease, a primary target is the ventral intermediate nucleus (VIM) for candidates for deep brain stimulation [34].

The deep brain stimulation system has four available parameters: stimulation amplitude, stimulation frequency, pulse width, and polarity [33]. The determination of an optimal parameter configuration is an inherent aspect of providing therapy through deep brain stimulation [35] [36]. Using current approaches, the optimization process can take on the order of several months [37].

Network Centric Therapy has been proposed as a viable strategy for optimizing the deep brain stimulation system parameters with the future goal of attaining real-time optimization. Central to this concept is the use of wearable and wireless systems for objectively quantifying tremor feedback with machine learning to distinguish between various parameter configuration scenarios. With wireless connectivity to Cloud computing resources a patient could be treated by expert clinicians effectively anywhere in the world [7].

\subsection{Quantification Techniques for Parkinson's Disease Tremor with Transition to Network Centric Therapy}

The ability to quantify the status of a neurodegenerative movement disorder, such as Parkinson's disease, is an inherent aspect for the ability to establish diagnosis and treatment strategy [7] [38]. Traditionally an ordinal scale technique is applied, for which a highly skilled clinician subjectively interprets an ordinal scale, such as the Unified Parkinson's Disease Rating Scale (UPDRS) [39] [40] [41] [42]. The reliability of the ordinal scale technique is a subject of contention [40] [43]. Furthermore, the translation between multiple established ordinal scale strategies has not been definitively established [40]. The use of wearable inertial sensors, such as accelerometers, has been proposed as an advancement beyond the inherently resource constrained ordinal scale technique [7] [39] [44].

Preliminary attempts to apply wearable accelerometer systems for evaluating intervention strategies for neurodegenerative movement disorders, such as Parkinson's disease, successfully demonstrated the potential of the inertial sensor. With these preliminary inertial sensor systems, the efficacy of various therapy 
interventions could be objectively assessed using quantified data [7] [39] [44]-[50]. The original data transfer methods have become effectively outmoded with the progressive evolution of the wearable and now wireless inertial sensor systems [7] [44] [51].

Preliminary wireless systems incorporated accelerometer systems secured by a glove or strap mechanism to the dorsum of the hand. The recorded acceleration dataset would be conveyed by local wireless connectivity to a laptop computer for subsequent post-processing [52] [53] [54]. Other similar evolutions involved wired connectivity of the inertial sensor system to a wrist mounted command module for wireless data transfer [55].

The nascent origins of Network Centric Therapy were demonstrated by LeMoyne et al. during 2010 with the application of a smartphone as a wearable and wireless accelerometer platform. The smartphone was mounted about the dorsum of the hand through a glove. A software application enabled the recording of the accelerometer signal, which could then be conveyed wirelessly as an email attachment to the Internet. During this preliminary demonstration the experimental and post-processing resources were situated on other sides of the continental United States of America [1].

Using the smartphone as a wearable and wireless inertial sensor system the efficacy of deep brain stimulation for the treatment of neurodegenerative movement disorders, such as Parkinson's disease and Essential tremor, has been successfully determined. The inertial sensor signal data were consolidated into a feature set through software automation for machine learning classification of deep brain stimulation system set to "On" and "Off" status. The machine learning algorithms achieved considerable classification accuracy to distinguish between deep brain stimulation system set to "On" and "Off" status for their respective movement disorder scenarios [6] [9] [56] [57] [58] [59].

There are many opportunities for improvement beyond the strategy of using the smartphone to quantify movement disorder tremor. The generational development of the smartphone has led to a more cumbersome device for mounting by glove at the dorsum of the hand. The smartphone is not intended for the acquisition of medical grade data. Also, instead of an actual Cloud computing environment, a provision email account was utilized [1] [6] [9] [56] [57] [58] [59].

The next evolutionary phase of Network Centric Therapy is represented by the development of the BioStamp nPoint, which constitutes a highly wearable and wireless system with a volumetric profile on the scale of a bandage that is more than ten times lighter than the standard smartphone. Furthermore, BioStamp $\mathrm{nPoint}$ is an FDA $510(\mathrm{k})$ cleared medical device for the acquisition of medical grade data. The BioStamp nPoint presents a segmented wireless strategy with local connectivity from the BioStamp nPoint equipped with inertial sensors, such as an accelerometer, to a more powerful wireless device, such as a smartphone. The more powerful wireless device conveys the BioStamp nPoint inertial sensor signal data to a Cloud computing resource for post-processing [10]. This 
strategy has been demonstrated and proposed as a subsequent evolution to the use of a smartphone as a wearable and wireless system for quantifying human movement [7] [8] [60] [61] [62]. The BioStamp nPoint is the recent evolution of conformal wearable and wireless systems that have been advocated and successfully demonstrated for acquiring quantified characteristics of movement disorders, such as Parkinson's disease [10]-[20].

The objective of the research endeavor is to demonstrate the preliminary Network Centric Therapy for the treatment of Parkinson's disease through deep brain stimulation. The BioStamp nPoint provides a highly wearable and wireless inertial sensor system mounted to the dorsum of the hand for quantifying deep brain stimulation set to "On" and "Off" status. The inertial sensor signal is consolidated into a feature set through software automation. Machine learning classification using the multilayer perceptron neural network attains classification accuracy to distinguish between the deep brain stimulation set to "On" and "Off" status for a subject with Parkinson's disease.

\section{Material and Methods}

Preliminary demonstration of Network Centric Therapy was achieved from the perspective of engineering proof of concept for the treatment of Parkinson's disease through a deep brain stimulation system, for which the efficacy of the treatment was established based on machine learning classification to distinguish between "On" and "Off" status of deep brain stimulation. The BioStamp nPoint provided the inherent aspects of Network Centric Therapy, as it constitutes a highly wearable and wireless inertial sensor system capable of providing an accelerometer signal with a volumetric profile on the scale of a bandage. The BioStamp nPoint acquires local connectivity to a device with broader wireless capability, such as a smartphone. The smartphone subsequently transmits the inertial sensor signal data wirelessly to a Cloud computing resource for post-processing anywhere in the world with sufficient Internet connectivity [10].

One subject (66-year-old female) diagnosed with Parkinson's disease in 2011 was selected while being administered bilateral subthalamic nucleus deep brain stimulation. Informed consent was established, and the experimental research was conducted at Allegheny General Hospital in conjunction with ethical clearance. The BioStamp nPoint was mounted about the dorsum of the hand through an adhesive medium, such that the BioStamp nPoint was symmetrically aligned in a longitudinal manner relatively to the third metacarpal (the aspect of the dorsum of the hand that is a collinear extension of the middle finger). This mounting strategy emulates the successful applications of wearable and wireless systems for objectively quantifying Parkinson's disease hand tremor [1] [6] [26] [54] [57] [58]. Figure 1 illustrates a representative mounting strategy of the BioStamp nPoint. Figure 2 presents BioStamp nPoint with the associated supporting apparatus for operation. Figure 3 provides the deep brain stimulation system clinician programmer, which is capable of controlling the "On" and "Off" mode settings. 


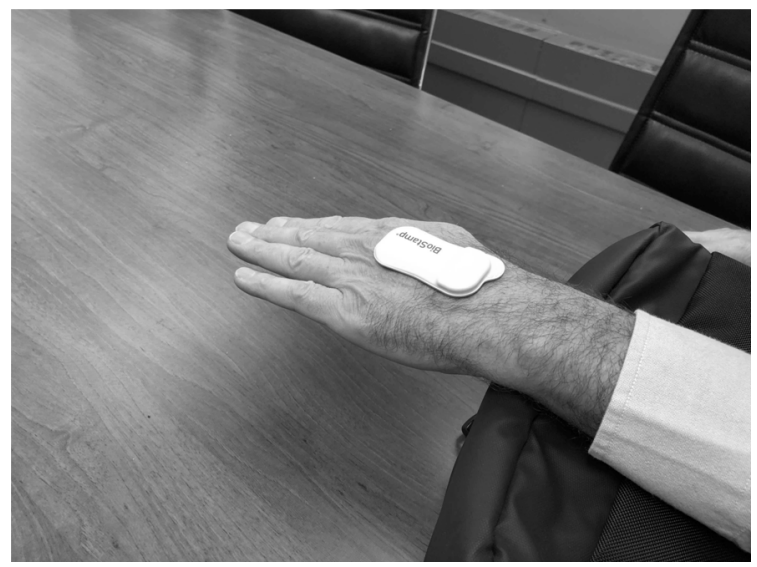

Figure 1. The BioStamp nPoint mounted about the dorsum of the hand symmetrically about the longitudinal aspect of the third metacarpal.

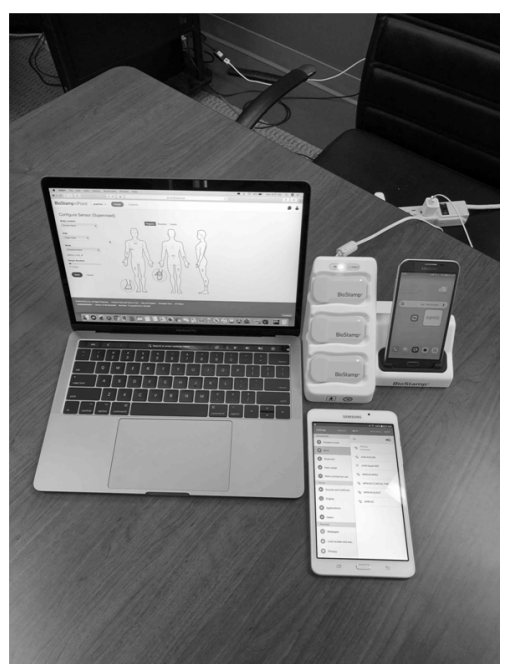

Figure 2. The BioStamp nPoint and supporting apparatus (computer, docking station, smartphone, and tablet).

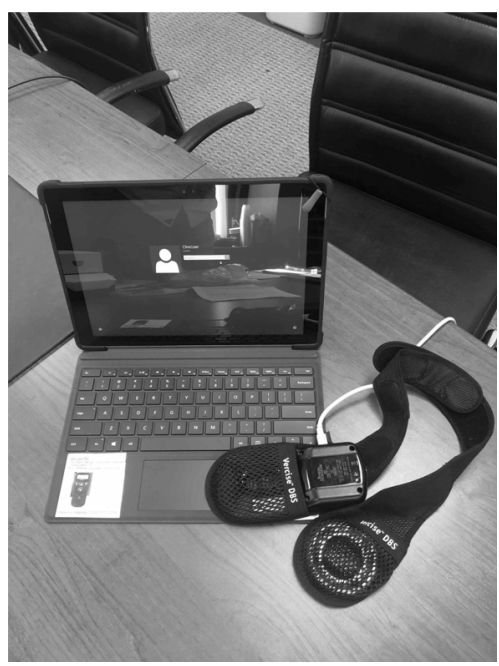

Figure 3. The deep brain stimulation system clinician programmer for commanding "On" and "Off" modes. 
Machine learning classification was conducted through the Waikato Environment for Knowledge Analysis (WEKA) [6] [9] [56] [57] [58] [59]. In particular the multilayer perceptron neural network was considered to be the optimal machine learning algorithm, and ten-fold cross validation was incorporated into the machine learning classification endeavor. The application of WEKA requires the development of an Attribute-Relation File Format (ARFF) based on the numeric attributes that compose the feature set [63] [64] [65].

The inertial signal data from the BioStamp nPoint was consolidated to develop the ARFF, which was based on the magnitude of the accelerometer signal. The ARFF was established through software automation enabled by Python. Based on previous successful machine learning applications involving wearable and wireless systems, the feature set was composed of the following attributes for the magnitude of the accelerometer signal maximum, minimum, mean, standard deviation, and coefficient of variation [6] [62] [66] [67] [68].

The experimental protocol involved five trials each spanning two seconds for the deep brain stimulation system set to "On" status and five trials each spanning two seconds with the deep brain stimulation system set to "Off" status. The subject's wrist was suspended beyond a support for the forearm for each respective trial. The BioStamp nPoint was set to $250 \mathrm{~Hz}$ for the sampling rate of the accelerometer signal with the recording set to a sufficient duration to provide two second intervals for each of the five trials. The experiment was conducted based on the following protocol:

1) Mount the BioStamp nPoint through an adhesive medium to the dorsum of the hand, such that the BioStamp nPoint is symmetrically and longitudinally oriented about the third metatarsal.

2) Orient the forearm of the subject relative to the support, such that the wrist is suspended beyond the support, ensuring that hand tremor is unimpaired.

3) Activate the recording process of the BioStamp nPoint for acquisition of the BioStamp nPoint accelerometer signal with pending upload to the Cloud computing resource.

4) Continue recording duration such that five trials spanning two seconds can be acquired with the deep brain stimulation system set to "On" status.

5) Repeat the same protocol for the deep brain stimulation system set to "Off" status.

\section{Results and Discussion}

\subsection{Results}

The hand tremor for the subject with Parkinson's disease is perceptivity disparate from an observational perspective regarding deep brain stimulation set to "On" status and "Off" status. The BioStamp nPoint enables quantification of the hand tremor for each respective scenario through inertial sensors, such as an accelerometer. The visualized quantification of the acceleration magnitude is readily determined. Figure 4 represents hand tremor acceleration magnitude for 
the subject with Parkinson's disease while the deep brain stimulation system is set to "On" status. Figure 5 illustrates the hand tremor acceleration magnitude with the deep brain stimulation system set to "Off" status.

Based on the observation of the inertial sensor signal data a feature set for the five instances of deep brain stimulation system set to "On" status and five instances of deep brain stimulation system set to "Off" status is consolidated through five attributes:

- maximum;

- minimum;

- mean;

- standard deviation;

- coefficient of variation.

These attributes back been successfully applied to an assortment of machine learning classification endeavors pertaining to the application of wearable and wireless inertial sensor systems [6] [62] [66] [67] [68].

WEKA consists of a considerable array of machine learning classification algorithms. The multilayer perceptron neural network was selected as the most suitable machine learning algorithm to distinguish between deep brain stimulation system set to "On" status and deep brain stimulation system set to "Off" status based on quantification of the inertial sensor signals derived from a wearable and wireless system. The acquired multilayer perceptron neural network is presented in Figure 6. A classification accuracy of $100 \%$ was achieved by the multilayer perceptron neural network for differentiating between deep brain stimulation set to "On" and "Off" status for a subject with Parkinson's disease.

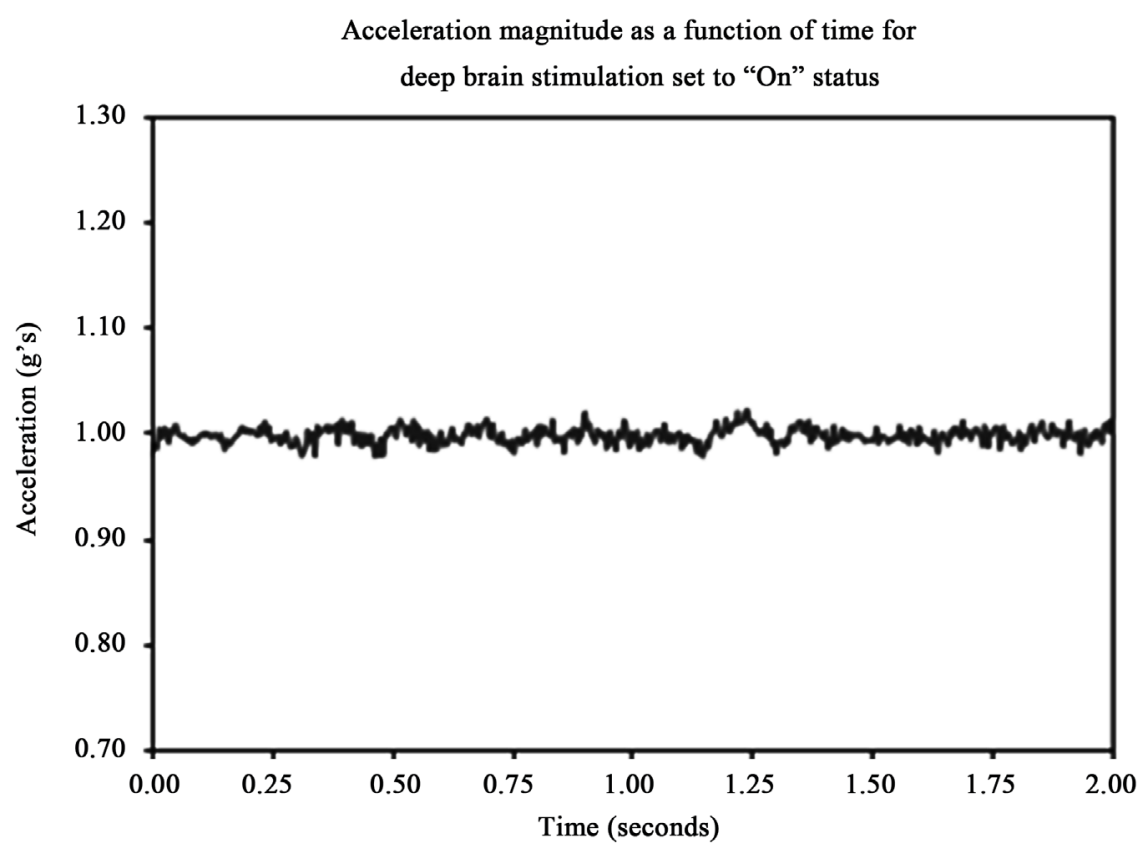

Figure 4. The acceleration magnitude of hand tremor for the Parkinson's disease subject with the deep brain stimulation system set to "On" status. 


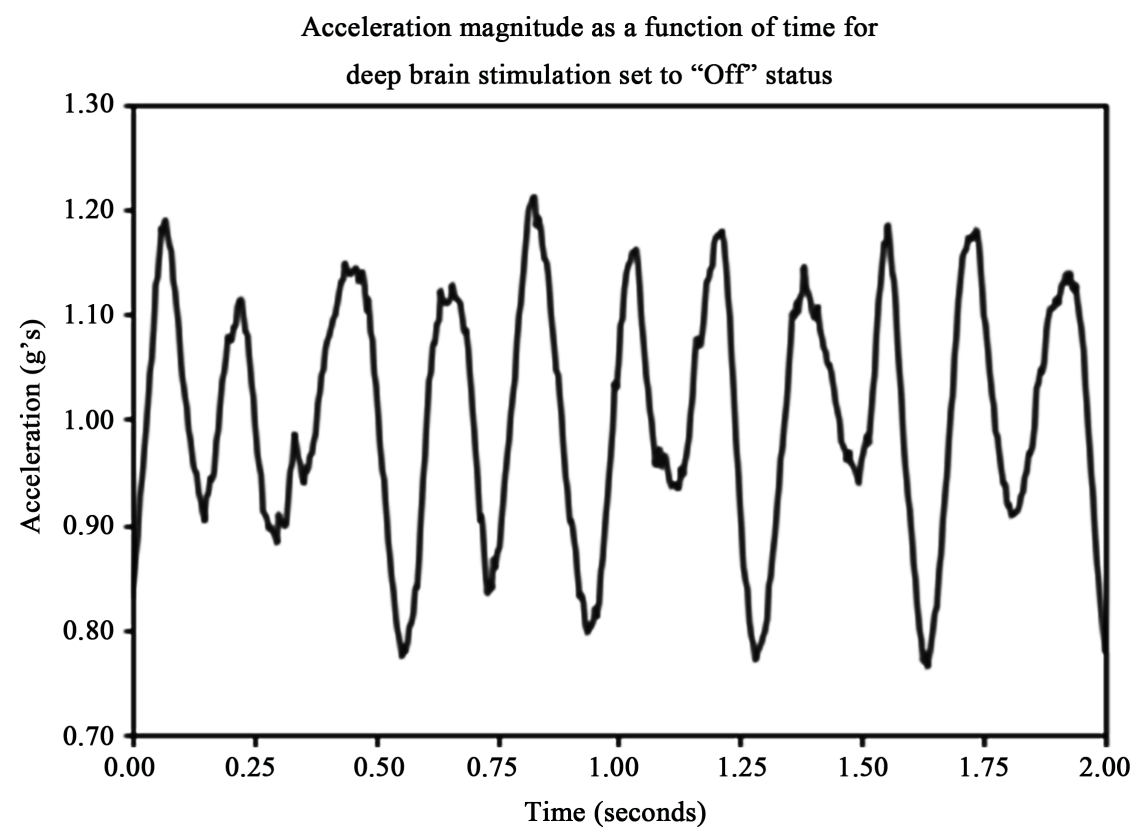

Figure 5. The acceleration magnitude of hand tremor for the Parkinson's disease subject with the deep brain stimulation system set to "Off" status.

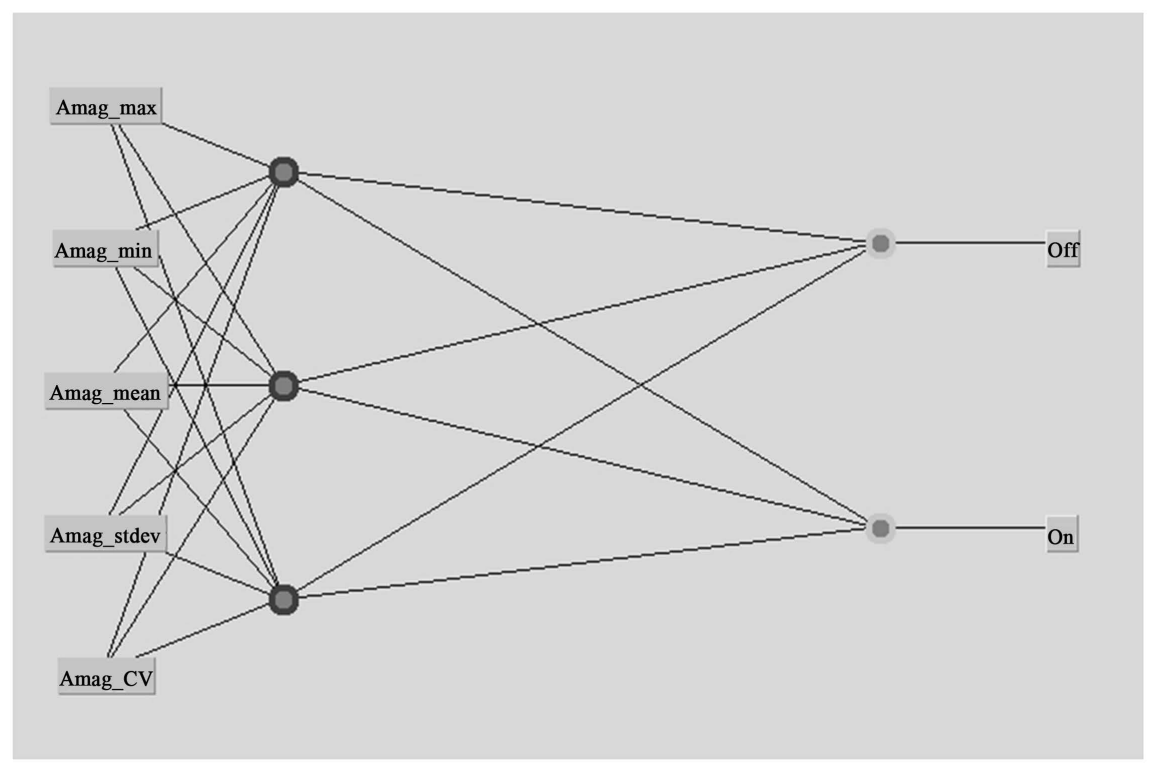

Figure 6. The multilayer perceptron neural network for distinguishing hand tremor for a subject with Parkinson's disease with respect to deep brain stimulation set to "On" and "Off" status based on a wearable and wireless inertial sensor system. The input layer consists of five feature set attributes (Amag_max: maximum of the acceleration magnitude, Amag_min: minimum of the acceleration magnitude, Amag_mean: mean of the acceleration magnitude, Amag_stdev: standard deviation of the acceleration magnitude, and Amag_CV: coefficient of variation of the acceleration magnitude).

\subsection{Discussion}

The BioStamp nPoint serves as a preliminary demonstration of the opportunity and potential of Network Centric Therapy, which represents a highly wearable 
and wireless inertial sensor system. A segmented wireless strategy is utilized, which locally transmits inertial sensor signal data to a device, such as a smartphone, for broader wireless connectivity to the Internet through access to Cloud computing resources. In fact, the BioStamp nPoint is effectively conformal about the dorsum of the hand, underscoring the inherent value of highly wearable and wireless systems for Network Centric Therapy.

With the inertial sensor signal acquired the data can be consolidated into a feature set for machine learning classification. The role of machine learning is envisioned as instrumental for augmenting the clinician's ability to rapidly converge upon an optimal series of parameter configuration for efficacious deep brain stimulation therapy. In particular, the Cloud computing storage of the inertial sensor signal data through wireless connectivity enables a skilled clinician to proactively optimize treatment strategy for a subject with a movement disorder, such as Parkinson's disease.

The achievements of the research objective elucidate perspective regarding future goals, such as automated real-time optimization of deep brain stimulation system parameter configurations. Machine learning and highly wearable and wireless systems that are effectively conformal and envisioned serve as inherent aspects of achieving the real-time automation of parameter configurations capability for deep brain stimulation systems for the treatment of movement disorders, such as Parkinson's disease. Another design requirement to consider is whether to conduct the machine learning classification at the Cloud computing resource level or intrinsic to the wearable and wireless systems, for which respective Network Centric Therapy architectures have been proposed [7] [69].

The selection of the appropriate machine learning algorithm is imperative depending on the selected Network Centric Therapy architecture. A wearable and wireless system would likely be equipped with substantially less processing capability than a Cloud computing resource, however the wireless transmission of an optimized parameter configuration would be essentially instantaneous. Previous research has addressed and contrasted the processing time to attain classification accuracy for an assortment of machine learning algorithms regarding the application of wearable and wireless systems for determining the efficacy of deep brain stimulation to ameliorate movement disorder tremor symptoms [9] [58] [59]. In summary, Network Centric Therapy with the integration of wearable and wireless systems with Cloud computing access, such as the BioStamp nPoint, deep brain stimulation, and machine learning are envisioned to provide a quantum leap for the treatment of movement disorders, such as Parkinson's disease.

\section{Conclusions}

Network Centric Therapy has been successfully demonstrated through the implementation of wearable and wireless systems and machine learning that utilizes the synergistic capabilities of Cloud computing for the treatment of Parkinson's disease through deep brain stimulation. The Biostamp nPoint represents a 
highly conformal wearable and wireless system with a profile on the order of a bandage that offers a considerable reduction in mass compared to other devices, such as a smartphone. This device achieves wireless access to a Cloud computing environment. The inherent characteristics of this conformal wearable and wireless system enable mounting to the dorsum of the hand by adhesive as opposed to a specialized glove used in previous experiments to secure a device, such as a smartphone. The BioStamp nPoint acquired accelerometer signal data to quantify the efficacy of a deep brain stimulation system for treating a subject with Parkinson's disease. The magnitude of the accelerometer signal was consolidated to a feature set through software automation using Python. A multilayer perceptron neural network machine learning algorithm achieved considerable classification accuracy to distinguish between deep brain stimulation set to "On" and "Off" status for the treatment of a subject with Parkinson's disease using the BioStamp nPoint, which is FDA cleared for the acquisition of medical grade data.

The association of these achievements infers the pathway to the progressive research, development, testing, and evaluation of Network Centric Therapy for the treatment of people with movement disorders. Network Centric Therapy represents a combination of the expansive opportunities enabled with conformal wearable and wireless systems with segmented access to a Cloud computing environment with machine learning to augment clinical situational awareness respective of the efficacy of the prescribed intervention strategy. Respective of deep brain stimulation, these capabilities are envisioned to provide a pathway for the optimization of deep brain stimulation parameters in a closed-loop context for people with Parkinson's disease.

\section{Acknowledgements}

Our research team would like to extend our gratitude to Allegheny General Hospital for making this contribution to the amelioration of movement disorder symptoms possible.

\section{Conflicts of Interest}

The authors declare no conflicts of interest regarding the publication of this paper.

\section{References}

[1] LeMoyne, R., Mastroianni, T., Cozza, M., Coroian, C. and Grundfest, W. (2010) Implementation of an iPhone for Characterizing Parkinson's Disease Tremor through a Wireless Accelerometer Application. Proceedings of the 32nd Annual International Conference of the IEEE EMBS, Buenos Aires, 31 August-4 September 2010, 4954-4958. https://doi.org/10.1109/IEMBS.2010.5627240

[2] LeMoyne, R. and Mastroianni, T. (2015) Chapter 23 Use of Smartphones and Portable Media Devices for Quantifying Human Movement Characteristics of Gait, Tendon Reflex Response, and Parkinson's Disease Hand Tremor. In: Rasooly, A. and Herold, K.E., Eds., Mobile Health Technologies. Methods and Protocols, Springer, New York, 335-358. https://doi.org/10.1007/978-1-4939-2172-0_23 
[3] LeMoyne, R. and Mastroianni, T. (2017) Chapter 1 Smartphone and Portable Media Device: A Novel Pathway toward the Diagnostic Characterization of Human Movement. In: Mohamudally, N., Ed., Smartphones from an Applied Research Perspective, InTech, Rijeka, 1-24. https://doi.org/10.5772/intechopen.69961

[4] LeMoyne, R. and Mastroianni, T. (2017) Chapter 6 Wearable and Wireless Gait Analysis Platforms: Smartphones and Portable Media Devices. In: Uttamchandani, D., Ed., Wireless MEMS Networks and Applications, Elsevier, New York, 129-152. https://doi.org/10.1016/B978-0-08-100449-4.00006-3

[5] LeMoyne, R. and Mastroianni, T. (2016) Telemedicine Perspectives for Wearable and Wireless Applications Serving the Domain of Neurorehabilitation and Movement Disorder Treatment. Telemedicine, SMGroup, Dover, 1-10.

[6] LeMoyne, R., Mastroianni, T., McCandless, C., Currivan, C., Whiting, D. and Tomycz, N. (2018) Implementation of a Smartphone as a Wearable and Wireless Accelerometer and Gyroscope Platform for Ascertaining Deep Brain Stimulation Treatment Efficacy of Parkinson's Disease through Machine Learning Classification. Advances in Parkinson's Disease, 7, 19-30.

[7] LeMoyne, R., Mastroianni, T., Whiting, D. and Tomycz, N. (2019) Wearable and Wireless Systems for Healthcare II. Springer, Singapore.

https://doi.org/10.1007/978-981-13-5808-1

[8] LeMoyne, R., Mastroianni, T., Whiting, D. and Tomycz, N. (2019) Chapter 7 Wearable and Wireless Systems with Internet Connectivity for Quantification of Parkinson's Disease and Essential Tremor Characteristics. In: Wearable and Wireless Systems for Healthcare II, Springer, Singapore, 79-97.

https://doi.org/10.1007/978-981-13-5808-1_7

[9] LeMoyne, R., Mastroianni, T., Whiting, D. and Tomycz, N. (2019) Chapter 9 Assessment of Machine Learning Classification Strategies for the Differentiation of Deep Brain Stimulation "On” and "Off” Status for Parkinson's Disease Using a Smartphone as a Wearable and Wireless Inertial Sensor for Quantified Feedback. In: Wearable and Wireless Systems for Healthcare II, Springer, Singapore, 113-126. https://doi.org/10.1007/978-981-13-5808-1_9

[10] MC10 Inc. https://www.mc10inc.com/our-products\#biostamp-npoint

[11] Jarchi, D., Pope, J., Lee, T.K., Tamjidi, L., Mirzaei, A. and Sanei, S. (2018) A Review on Accelerometry-Based Gait Analysis and Emerging Clinical Applications. IEEE Reviews in Biomedical Engineering, 11, 177-194. https://doi.org/10.1109/RBME.2018.2807182

[12] Dinesh, K., Xiong, M., Adams, J., Dorsey, R. and Sharma, G. (2016) Signal Analysis for Detecting Motor Symptoms in Parkinson's and Huntington's Disease Using Multiple Body-Affixed Sensors: A Pilot Study. IEEE Western New York Image and Signal Processing Workshop (WNYISPW), Rochester, 18 November 2016, 1-5. https://doi.org/10.1109/WNYIPW.2016.7904834

[13] Lonini, L., Dai, A., Shawen, N., Simuni, T., Poon, C., Shimanovich, L., Daeschler, M., Ghaffari, R., Rogers, J.A. and Jayaraman, A. (2018) Wearable Sensors for Parkinson's Disease: Which Data Are Worth Collecting for Training Symptom Detection Models. NPJ Digital Medicine, 1, 1-8. https://doi.org/10.1038/s41746-018-0071-z

[14] Adams, J.L., Dinesh, K., Xiong, M., Tarolli, C.G., Sharma, S., Sheth, N., Aranyosi, A.J., Zhu, W., Goldenthal, S., Biglan, K.M. and Dorsey, E.R. (2017) Multiple Wearable Sensors in Parkinson and Huntington Disease Individuals: A Pilot Study in Clinic and at Home. Digital Biomarkers, 1, 52-63. 
https://doi.org/10.1159/000479018

[15] Thorp, J.E., Adamczyk, P.G., Ploeg, H.L. and Pickett, K.A. (2018) Monitoring Motor Symptoms during Activities of Daily Living in Individuals with Parkinson's Disease. Frontiers in Neurology, 9, 1036. https://doi.org/10.3389/fneur.2018.01036

[16] Wright, J.M., Regele, O.B., Kourtis, L.C., Pszenny, S.M., Sirkar, R., Kovalchick, C. and Jones, G.B. (2017) Evolution of the Digital Biomarker Ecosystem. Digital Medicine, 3, 154-163. https://doi.org/10.4103/digm.digm_35_17

[17] Gibney, E. (2015) The Body Electric. Nature, 528, 26-28. https://doi.org/10.1038/528026a

[18] Sen-Gupta, E., Wright, D.E., Caccese, J.W., Wright Jr., J.A., Jortberg, E., Bhatkar, V., Ceruolo, M., Ghaffari, R., Clason, D.L., Maynard, J.P. and Combs, A.H. (2019) A Pivotal Study to Validate the Performance of a Novel Wearable Sensor and System for Biometric Monitoring in Clinical and Remote Environments. Digital Biomarkers, 3, 1-13. https://doi.org/10.1159/000493642

[19] Mombers, C., Legako, K. and Gilchrist, A. (2016) Identifying Medical Wearables and Sensor Technologies that Deliver Data on Clinical Endpoints. British Journal of Clinical Pharmacology, 81, 196-198. https://doi.org/10.1111/bcp.12818

[20] Kraemer, F.A., Braten, A.E., Tamkittikhun, N. and Palma, D. (2017) Fog Computing in Healthcare: A Review and Discussion. IEEE Access, 5, 9206-9222. https://doi.org/10.1109/ACCESS.2017.2704100

[21] Kandel, E.R., Schwartz, J.H. and Jessell, T.M. (2000) Chapter 43 Principles of Neural Science. McGraw-Hill, New York.

[22] Seeley, R.R., Stephens, T.D. and Tate, P. (2003) Chapter 14 Anatomy and Physiology. McGraw-Hill, Boston.

[23] Bickley, L.S. and Szilagyi, P.G. (2003) Chapter 16 Bates' Guide to Physical Examination and History Taking. Lippincott Williams and Wilkins, Philadelphia.

[24] Diamond, M.C., Scheibel, A.B. and Elson, L.M. (1985) Chapter 5 the Human Brain Coloring Book. Harper Perennial, New York.

[25] Nolte, J. and Sundsten, J.W. (2002) Chapter 19 the Human Brain: An Introduction to Its Functional Anatomy. Mosby, St. Louis.

[26] LeMoyne, R. (2013) Wearable and Wireless Accelerometer Systems for Monitoring Parkinson's Disease Patients-A Perspective Review. Advances in Parkinson's Disease, 2, 113-115. https://doi.org/10.4236/apd.2013.24021

[27] Giller, C.A., Dewey, R.B., Ginsburg, M.I., Mendelsohn, D.B. and Berk, A.M. (1998) Stereotactic Pallidotomy and Thalamotomy Using Individual Variations of Anatomic Landmarks for Localization. Neurosurgery, 42, 56-65. https://doi.org/10.1097/00006123-199801000-00011

[28] Niranjan, A., Kondziolka, D., Baser, S., Heyman, R. and Lunsford, L.D. (2000) Functional Outcomes after Gamma Knife Thalamotomy for Essential Tremor and MS-Related Tremor. Neurology, 55, 443-446. https://doi.org/10.1212/WNL.55.3.443

[29] Young, R.F., Jacques, S., Mark, R., Kopyov, O., Copcutt, B., Posewitz, A. and Li, F. (2000) Gamma Knife Thalamotomy for Treatment of Tremor: Long-Term Results. Journal of Neurosurgery, 93, 128-135. https://doi.org/10.3171/jns.2000.93.supplement_3.0128

[30] Williams, R. (2010) Alim-Louis Benabid: Stimulation and Serendipity. Lancet Neurology, 9, 1152. https://doi.org/10.1016/S1474-4422(10)70291-X

[31] Miocinovic, S., Somayajula, S., Chitnis, S. and Vitek, J.L. (2013) History, Applica- 
tions, and Mechanisms of Deep Brain Stimulation. JAMA Neurology, 70, 163-171. https://doi.org/10.1001/2013.jamaneurol.45

[32] Benabid, A.L., Pollak, P., Louveau, A., Henry, S. and de Rougemont, J. (1987) Combined (Thalamotomy and Stimulation) Stereotactic Surgery of the VIM Thalamic Nucleus for Bilateral Parkinson Disease. Applied Neurophysiology, 50, 344-346. https://doi.org/10.1159/000100803

[33] Amon, A. and Alesch, F. (2017) Systems for Deep Brain Stimulation: Review of Technical Features. Journal of Neural Transmission, 124, 1083-1091. https://doi.org/10.1007/s00702-017-1751-6

[34] Yu, H. and Neimat, J.S. (2008) The Treatment of Movement Disorders by Deep Brain Stimulation. Neurotherapeutics, 5, 26-36. https://doi.org/10.1016/j.nurt.2007.10.072

[35] Volkmann, J., Moro, E. and Pahwa, R. (2006) Basic Algorithms for the Programming of Deep Brain Stimulation in Parkinson's Disease. Movement Disorders, 21, S284-S289. https://doi.org/10.1002/mds.20961

[36] Isaias, I.U. and Tagliati, M. (2008) Chapter 20 Deep Brain Stimulation Programming for Movement Disorders. In: Tarsy, D., Vitek, J.L., Starr, P.A. and Okun, M.S., Eds., Deep Brain Stimulation in Neurological and Psychiatric Disorders, Springer, New York, 361-397. https://doi.org/10.1007/978-1-59745-360-8_20

[37] Pretto, T. (2007) Deep Brain Stimulation. Neurologist, 13, 103-104. https://doi.org/10.1097/01.nrl.0000258304.16124.e5

[38] LeMoyne, R., Mastroianni, T., Whiting, D. and Tomycz, N. (2019) Chapter 3 Traditional Ordinal Strategies for Establishing the Severity and Status of Movement Disorders, Such as Parkinson's Disease and Essential Tremor. In: Wearable and Wireless Systems for Healthcare II, Springer, Singapore, 25-36. https://doi.org/10.1007/978-981-13-5808-1_3

[39] LeMoyne, R., Coroian, C., Mastroianni, T., Opalinski, P., Cozza, M. and Grundfest, W. (2009) Chapter 10 the Merits of Artificial Proprioception, with Applications in Biofeedback Gait Rehabilitation Concepts and Movement Disorder Characterization. In: Barros de Mello, C.A., Ed., Biomedical Engineering, InTech, Vienna, 165-198. https://doi.org/10.5772/7883

[40] Ramaker, C., Marinus, J., Stiggelbout, A.M. and Van Hilten, B.J. (2002) Systematic Evaluation of Rating Scales for Impairment and Disability in Parkinson's Disease. Movement Disorders, 17, 867-876. https://doi.org/10.1002/mds.10248

[41] Goetz, C.G., Stebbins, G.T., Chmura, T.A., Fahn, S., Poewe, W. and Tanner, C.M. (2010) Teaching Program for the Movement Disorder Society-Sponsored Revision of the Unified Parkinson's Disease Rating Scale: (MDS-UPDRS). Movement Disorders, 25, 1190-1194. https://doi.org/10.1002/mds.23096

[42] Movement Disorder Society Task Force on Rating Scales for Parkinson's Disease (2003) The Unified Parkinson's Disease Rating Scale (UPDRS): Status and Recommendations. Movement Disorders, 18, 738-750. https://doi.org/10.1002/mds.10473

[43] Post, B., Merkus, M.P., de Bie, R., de Haan, R.J. and Speelman, J.D. (2005) Unified Parkinson's Disease Rating Scale Motor Examination: Are Ratings of Nurses, Residents in Neurology, and Movement Disorders Specialists Interchangeable? Movement Disorders, 20, 1577-1584. https://doi.org/10.1002/mds.20640

[44] LeMoyne, R., Mastroianni, T., Whiting, D. and Tomycz, N. (2019) Chapter 6 Preliminary Wearable and Locally Wireless Systems for Quantification of Parkinson's Disease and Essential Tremor Characteristics. In: Wearable and Wireless Systems for Healthcare II, Springer, Singapore, 65-78. 
https://doi.org/10.1007/978-981-13-5808-1_6

[45] Schrag, A., Schelosky, L., Scholz, U. and Poewe, W. (1999) Reduction of Parkinsonian Signs in Patients with Parkinson's Disease by Dopaminergic versus Anticholinergic Single-Dose Challenges. Movement Disorders, 14, 252-255. https://doi.org/10.1002/1531-8257(199903)14:2<252::AID-MDS1009>3.0.CO;2-N

[46] Keijsers, N.L., Horstink, M.W., van Hilten, J.J., Hoff, J.I. and Gielen, C.C. (2000) Detection and Assessment of the Severity of Levodopa-Induced Dyskinesia in $\mathrm{Pa}$ tients with Parkinson's Disease by Neural Networks. Movement Disorders, 15, 1104-1111. https://doi.org/10.1002/1531-8257(200011)15:6<1104::AID-MDS1007>3.0.CO;2-E

[47] Keijsers, N.L., Horstink, M.W. and Gielen, S.C. (2006) Ambulatory Motor Assessment in Parkinson's Disease. Movement Disorders, 21, 34-44.

https://doi.org/10.1002/mds.20633

[48] Gurevich, T.Y., Shabtai, H., Korczyn, A.D., Simon, E.S. and Giladi, N. (2006) Effect of Rivastigmine on Tremor in Patients with Parkinson's Disease and Dementia. Movement Disorders, 21, 1663-1666. https://doi.org/10.1002/mds.20971

[49] Obwegeser, A.A., Uitti, R.J., Witte, R.J., Lucas, J.A., Turk, M.F. and Wharen Jr., R.E. (2001) Quantitative and Qualitative Outcome Measures after Thalamic Deep Brain Stimulation to Treat Disabling Tremors. Neurosurgery, 48, 274-284. https://doi.org/10.1227/00006123-200102000-00004

[50] Kumru, H., Summerfield, C., Valldeoriola, F. and Valls-Solé, J. (2004) Effects of Subthalamic Nucleus Stimulation on Characteristics of EMG Activity Underlying Reaction Time in Parkinson's Disease. Movement Disorders, 19, 94-100. https://doi.org/10.1002/mds.10638

[51] Patel, S., Park, H., Bonato, P., Chan, L. and Rodgers, M. (2012) A Review of Wearable Sensors and Systems with Application in Rehabilitation. Journal of NeuroEngineering and Rehabilitation, 9, 1-17. https://doi.org/10.1186/1743-0003-9-21

[52] LeMoyne, R., Coroian, C. and Mastroianni, T. (2008) 3D Wireless Accelerometer Characterization of Parkinson's Disease Status. Proceedings of the Plasticity and Repair in Neurodegenerative Disorders, Lake Arrowhead, May 2008.

[53] LeMoyne, R., Coroian, C. and Mastroianni, T. (2009) Quantification of Parkinson's Disease Characteristics Using Wireless Accelerometers. Proceeding of the International Conference on Complex Medical Engineering (CME-2009) of the IEEE/ICME, Tempe, 9-11 April 2009, 1-5. https://doi.org/10.1109/ICCME.2009.4906657

[54] LeMoyne, R., Mastroianni, T. and Grundfest, W. (2013) Wireless Accelerometer Configuration for Monitoring Parkinson's Disease Hand Tremor. Advances in Parkinson's Disease, 2, 62-67. https://doi.org/10.4236/apd.2013.22012

[55] Giuffrida, J.P., Riley, D.E., Maddux, B.N. and Heldman, D.A. (2009) Clinically Deployable Kinesia Technology for Automated Tremor Assessment. Movement Disorders, 24, 723-730. https://doi.org/10.1002/mds.22445

[56] LeMoyne, R., Tomycz, N., Mastroianni, T., McCandless, C., Cozza, M. and Peduto, D. (2015) Implementation of a Smartphone Wireless Accelerometer Platform for Establishing Deep Brain Stimulation Treatment Efficacy of Essential Tremor with Machine Learning. Proceedings of the 37 th Annual International Conference of the IEEE EMBS, Milan, 25-29 August 2015, 6772-6775. https://doi.org/10.1109/EMBC.2015.7319948

[57] LeMoyne, R., Mastroianni, T., Tomycz, N., Whiting, D., Oh, M., McCandless, C., Currivan, C. and Peduto, D. (2017) Implementation of a Multilayer Perceptron Neural Network for Classifying Deep Brain Stimulation in "On" and "Off” Modes 
through a Smartphone Representing a Wearable and Wireless Sensor Application. Proceedings of the 47 th Annual Meeting of the Society for Neuroscience, Washington DC, 11-15 November 2017.

[58] LeMoyne, R., Mastroianni, T., McCandless, C., Currivan, C., Whiting, D. and Tomycz, N. (2018) Implementation of a Smartphone as a Wearable and Wireless Inertial Sensor Platform for Determining Efficacy of Deep Brain Stimulation for Parkinson's Disease Tremor through Machine Learning. Proceedings of the 48th Annual Meeting of the Society for Neuroscience (Nanosymposium), San Diego, 3-7 November 2018.

[59] LeMoyne, R., Mastroianni, T., McCandless, C., Whiting, D. and Tomycz, N. (2019) Evaluation of Machine Learning Algorithms for Classifying Deep Brain Stimulation Respective of "On" and “Off” Status. Proceedings of the 9 th International IEEE/EMBS Conference on Neural Engineering (NER), San Francisco, 20-23 March 2019, 483-488. https://doi.org/10.1109/NER.2019.8717095

[60] LeMoyne, R. and Mastroianni, T. (2018) Wearable and Wireless Systems for Healthcare I. Springer, Singapore. https://doi.org/10.1007/978-981-10-5684-0

[61] LeMoyne, R. and Mastroianni, T. (2018) Chapter 7 Bluetooth Inertial Sensors for Gait and Reflex Response Quantification with Perspectives Regarding Cloud Computing and the Internet of Things. In: Wearable and Wireless Systems for Healthcare I, Springer, Singapore, 95-103. https://doi.org/10.1007/978-981-10-5684-0_7

[62] LeMoyne, R., Heerinckx, F., Aranca, T., De Jager, R., Zesiewicz, T. and Saal, H.J. (2016) Wearable Body and Wireless Inertial Sensors for Machine Learning Classification of Gait for People with Friedreich's Ataxia. Proceedings of the 13 th International Conference on Wearable and Implantable Body Sensor Networks, San Francisco, 14-17 June 2016, 147-151. https://doi.org/10.1109/BSN.2016.7516249

[63] Hall, M., Frank, E., Holmes, G., Pfahringer, B., Reutemann, P. and Witten, I.H. (2009) The WEKA Data Mining Software: An Update. ACM SIGKDD Explorations Newsletter, 11, 10-18. https://doi.org/10.1145/1656274.1656278

[64] Witten, I.H., Frank, E. and Hall, M.A. (2011) Data Mining: Practical Machine Learning Tools and Techniques. Morgan Kaufmann, Burlington.

[65] WEKA. http://www.cs.waikato.ac.nz/ ml/weka

[66] LeMoyne, R. and Mastroianni, T. (2017) Virtual Proprioception for Eccentric Training. Proceedings of the 39th Annual International Conference of the IEEE EMBS, Jeju Island, 11-15 July 2017, 4557-4561. https://doi.org/10.1109/EMBC.2017.8037870

[67] LeMoyne, R. and Mastroianni, T. (2017) Wireless Gyroscope Platform Enabled by a Portable Media Device for Quantifying Wobble Board Therapy. Proceedings of the 39th Annual International Conference of the IEEE EMBS, Jeju Island, 11-15 July 2017, 2662-2666. https://doi.org/10.1109/EMBC.2017.8037405

[68] LeMoyne, R. and Mastroianni, T. (2016) Implementation of a Smartphone as a Wireless Gyroscope Platform for Quantifying Reduced Arm Swing in Hemiplegic Gait with Machine Learning Classification by Multilayer Perceptron Neural Network. Proceedings of the 38 th Annual International Conference of the IEEE EMBS, Orlando, 16-20 August 2016, 2626-2630. https://doi.org/10.1109/EMBC.2016.7591269

[69] LeMoyne, R., Mastroianni, T., Whiting, D. and Tomycz, N. (2019) Chapter 10 New Perspectives for Network Centric Therapy for the Treatment of Parkinson's Disease and Essential Tremor. In: Wearable and Wireless Systems for Healthcare II, Springer, Singapore, 127-128. https://doi.org/10.1007/978-981-13-5808-1_10 